\title{
QUANTUM MECHANICS AND CONSCIOUSNESS
}

\author{
Friedrich Beck, Institut für Kernphysik, Technische Hochschule Darmstadt, \\ D-64289 Darmstadt, Germany.Email: beck@crunch.ikp.physik.th-darmstadt.de
}

\begin{abstract}
The first issue of $J C S$ published an interview with Roger Penrose on his recent book Shadows of the Mind: A Search for the Missing Science of Consciousness (Clark, 1994). In it Professor Penrose, among other subjects, presented his views on the role of quantum mechanics on our way towards a better understanding of brain functioning and its relation to consciousness. In this note we comment on some aspects of his reasoning.
\end{abstract}

\section{Quantum Aspects of Brain Activity}

In recent years there has been increasing interest in the question of whether quantum processes could play a decisive role in our basic understanding of brain activity. In a recent conference in Tucson, ${ }^{1}$ a whole double-session was devoted to the discussion of 'Quantum Theory and Consciousness'. In these discussions, however, the main emphasis has been on the question of whether the indeterminacy of quantum mechanics, especially in the course of the measuring process, can open a door for the action of a conscious will upon the physiological functioning of the brain. Very little is normally said about the concrete locus in the complex brain structure where quantum processes could effectively enter. It should be emphasized, however, that the whole question has these two aspects which should be clearly separated. One aspect is the epistemological one which is related to the interpretation of quantum mechanics, or even to an extension of the present theory, as Penrose seems to suggest in the interview; and it is admittedly of a speculative and hypothetical character. The other aspect is the search for a better understanding of the mechanism of synaptic action and its relation to microscopic or, in turn, large-scale coherent actions where quantum processes are important in the realm of present-day quantum theory. This latter question is certainly open to experimental and theoretical research in contemporary brain physiology, without the speculative problems of the first one.

A contribution to the second aspect has been presented by the author, in collaboration with John C. Eccles (Beck \& Eccles, 1992). The physiological basis was the process of exocytosis of transmitter substance at the synapses of the neocortex. It had been shown by careful statistical analysis, mainly performed by Sayer, Redman and Andersen (1989), that exocytosis is a process which responds to a nerve impulse with a probability much less than unity. By attributing this result to a quantum process we could show, by rather general quantum statistical considerations, that a trigger mechanism is needed that controls exocytosis by a microsite action involving at most the motion of one, or a few, hydrogen atoms in the membrane. A model was set up that attributes the trigger to the quantum-mechanical tunnelling of a quasi particle representing the micro site. It was furthermore argued that by long-range interactions within a larger area of the microsite a coherent quantum state could result, as is well known from macroscopically-ordered quantum systems.

A qualitative numerical estimate of our tunnelling model leads to reasonable numbers for the dynamic constituents and for the exocytosis probability as analysed by Redman (1990). The relaxation time of the trigger, however, came out in the femtosecond region, a time scale that is normally not reached in membrane physiological measurements: these

1 'Toward a Scientific Basis for Consciousness', Tucson, AZ, April 1994. 
are in the millisecond range. Because of this our tunnelling model, which was established in 1992, had at that time no direct experimental backing, except the (indirect) measurements of the exocytosis probability at a single synapse (Redman, 1990). Since that time there has been increasing experimental (Johansson and Århem, 1994) and theoretical (Lee, 1992) evidence that the critical structure for opening a membrane channel is indeed very small. In another context, namely for the bacterial photosynthesis reaction, ultrashort-time spectroscopy has shown that electron transfer processes are responsible for the charge separating transport through the membrane (Ogrodnik et al., 1988; Vos, 1993). Since nature uses successful processes over and over again in a variety of physiologically similar situations, this latter observation could have some bearing for the membrane trigger of synaptic exocytosis, and it could also stimulate short-time spectroscopy work in this area.

Several of these aspects have been addressed by Penrose in his interview, unfortunately without mentioning the original contribution of the author and John C. Eccles, though he acknowledges that he took part in a discussion about Eccles' new book (1994) during the Tucson conference. However, chapter nine of this book is a reprint of our earlier work (Beck and Eccles, 1992). The whole concept of the 'microtubule hypothesis', as being a main issue of the Tucson conference also - and by now adopted by Penrose as the process 'where quantum effects work their magic' — is no more than a membrane-physiological proposal for the trigger as introduced in our 1992 work.

\section{Quantum Aspects of Consciousness}

As pointed out in the first section, the relation between quantum processes and consciousness is still of a highly speculative and hypothetical character, and as such is controversial. One of the first authors who established a possible connection in this direction was E.P. Wigner (1967) who pointed out the principal inconsistency in understanding the measuring process in quantum mechanics in an objective, observer-independent way. Other developments have led in two different directions. One is the attempt to reinterpret orthodox quantum mechanics as, for example, in Everett's 'many worlds interpretation' (1957). The other claims that quantum mechanics has to be amended by a new mechanism of 'state collapse' to give measurements on quantum systems an objective basis (see Bell, 1987). Penrose also proposes this view. The question, however, in which form these developments could lead to a better understanding of the relation between consciousness and the brain, remains completely open; and it is, of course, independent of the physiological mechanisms discussed in the previous section. Penrose, having recently adopted the microtubule hypothesis, is addressing the physiological, not the epistemological aspect of the mind-brain problem.

In our work we presented one hypothesis (due to J.C. Eccles), showing how mental processes, such as for example intentions, could influence the brain. The important issue there was the assumption that selection of a quantum amplitude out of a superposition of different states (in our case the 'left' and 'right' partial amplitudes of the tunnelling wave function) does not violate physical conservation laws, and by this allows the control of brain processes without interfering with the dynamics. This, in our opinion, is a crucial point as to why the 'quantum brain' is different from the 'classical brain', where functioning is based on the deterministic and causal classical dynamics. In view of the fact that all this is very clearly expressed in chapters nine and ten of Eccles (1994), Penrose's statement, '[a]lthough I am not quite sure what Sir John Eccles does really think', seems rather ill-informed. Of course at this stage our proposal, like others, remains 
a hypothesis, left open to the question of whether it could ever be substantiated by experimental studies as, for example, in behavioural investigations. We feel, however, that this hypothesis, based on dualism, is by no means less convincing than other attempts to incorporate consciousness into the understanding of the human brain, a question which seems to be one of the most pressing and basic mysteries of brain research.

\section{References}

Beck, F. and Eccles, J.C. (1992), 'Quantum aspects of brain activity and the role of consciousness', Proc. Natl. Acad. Sci. USA, 89, p. 11357-61.

Bell, J.S. (1987), Speakable and Unspeakable in Quantum Mechanics (Cambridge: Cambridge University Press).

Clark, J. (1994), interview with Roger Penrose, J. Consciousness Studies, 1 (1), pp. 17-24.

Eccles, J.C. (1994), How the Self Controls its Brain (Berlin, Heidelberg \& NewYork: SpringerVerlag).

Everett, H. (1957), “"Relative state” formulation of quantum mechanics', Rev. Mod. Phys., 29, pp. 454-62.

Johansson, S. and Århem, P. (1994), 'Single-channel currents trigger action potentials in small cultured hippocampal neurons', Proc. Natl. Acad. Sci. USA, 91, p. 1761-5.

Lee, C.-Y. (1992), 'A possible biological role of the electron transfer between tyrosine and tryptophan. Gating of ion channels', FEBS Lett., 299, pp. 119-23.

Ogrodnik, A. et al. (1988), 'Determination of free energies in reaction centers of Rb. spaeroids', Biochim. Biophys. Acta, 936, pp. 361-71.

Redman, S.J. (1990), 'Quantal analysis of synaptic potentials in neurons of the central nerve system', Physiol. Rev.,70, pp. 165-98.

Sayer, R.J., Redman, S.J. and Andersen, P. (1989), 'Amplitude fluctuations in small EPSPs recorded from AC1 pyramidal cells in guinea pig hippocampal slice', J. Neurosci., 9, pp. 840-50.

Vos, M.H. (1993), 'Visualization of coherent nuclear motion in a membrane protein by femtosecond spectroscopy', Nature, 363, pp. 320-5.

Wigner, E.P. (1967), 'Remarks on the mind-body question', in Symmetries and Reflections (Bloomington, IN: Indiana University Press), p. 153. 